\title{
Impacts du changement climatique sur la croissance et le développement du blé en France
}

\author{
Quelles solutions et quelles actions à développer ?
}

\author{
Philippe GATE ${ }^{1}$ \\ Anne BLONDLOT ${ }^{1}$ \\ David GOUACHE ${ }^{1}$ \\ Olivier DEUDON ${ }^{1}$ \\ Laurent VIGNIER ${ }^{2}$ \\ ${ }^{1}$ Pôle Valorisations de l'Ecophysiologie, \\ ARVALIS - Institut du végétal, \\ domaine INRA-GEVES, La Minière, \\ 78280 Guyancourt, France \\ $<$ p.gate@arvalisinstitutduvegetal.fr> \\ ${ }^{2}$ EARL Rouillot
}

\begin{abstract}
Knowledge acquired over the paste decades on wheat physiology allows for an estimation of the impacts of key climatic factors on wheat physiology, yield components, as well as a quantification of the main climatic risks for the crop. Using historical weather data sets starting in 1955 from a large number of weather stations, we have concluded that a certain number of climatic risks have changed through comparison of two sequences: 1955-1980 and 1981-2005. Despite a significant anticipation of phenological stages, number of grains per $\mathrm{m}^{2}$ has been significantly affected by an incresase in water stress during stem extension; also, kernel weight is more strongly penalized through the joint effects of increased drought and especially increased heat stress during grain filling. These tendancies vary geographically. Certain regions have been affected early on (mediteranean region), whereas others are almost untouched as yet (western maritime front). Studies using projected future weather data validates this tendancy and allows its extrapolation. We propose adaptation pathways through escape based on varietal earliness and identify new phenological ideotypes and modes of growth better adapted to future risk. As a complement, the interest of selecting for cultivars tolerant to abiotic stresses is put forward, especially cultivars efficient with regards to water use and resistant to high temperatures. For these aspects, simple diagnostic methods and characterization tools are given. Finally, a preliminary analysis of the adaptation of cropping practices (sowing, nitrogen fertilization, lodging control) is initiated.
\end{abstract}

Key words: wheat, climate change, phenology, yield, adaptation

Dans un premier temps, notre travail a donc été de démontrer si effectivement, vis-à-vis de la culture du blé, les risques climatiques ont évolué ou non.

Pour cela, l'étude s'est focalisée sur les paramètres climatiques susceptibles d'avoir été modifiés par le changement climatique (température et disponibilité en eau) en évaluant leurs impacts sur la phénologie et le rendement : - chiffrage de l'anticipation des stades clés du développement ;

- avec cette anticipation éventuelle, diagnostic de l'évolution des risques climatiques intervenant à des stades sensibles;

- évolution des impacts climatiques sur deux composantes du rendement, le nombre de grains par $\mathrm{m}^{2}$ et le poids des grains.

Une telle démarche permet d'entrevoir si l'anticipation naturelle des stades se traduit ou non par un effet de compensation suffisant. En cas d'évitement naturel insuffisant, est-il possible $d^{\prime}$ envisager de nouvelles combinaisons date de semis - précocité variétale, ou faudra-il concevoir des variétés ayant de nouveaux profils phénologiques ? La stratégie d'évitement doitelle être accompagnée par une recherche de variétés plus tolérantes aux stress abiotiques?
Sur ce point, existe-t-il des méthodes simples de caractérisation variétale?

Enfin, pour d'autres techniques et interventions culturales, comme l'implantation, la protection contre la verse, la gestion de la fertilisation azotée, est-il justifié de les adapter à court terme?

Pour ces différents points, nous essayons d'apporter des éléments de réflexion et proposons des solutions adaptatives.

\section{Matériel et méthodes}

\section{Analyse des risques climatiques}

\section{Données météorologiques}

On se propose de chiffrer les conséquences de l'évolution climatique en analysant les données météorologiques historiques de 59 stations bien réparties dans les différents bassins de production, à l'échelle de la France.

Pour caractériser l'évolution du climat, nous avons mis la priorité sur l'analyse des données météorologiques historiques plutôt que d'utiliser en premier lieu les données prévisionnelles simulées, actuellement plus précises pour un futur trop lointain (à partir de 2070). 
Une première analyse consiste à comparer 2 séries chronologiques de taille égale: la première allant de 1955 à 1980 et la deuxième de 1981 à 2005. Ces résultats sont enrichis par une deuxième expertise consistant à identifier les évolutions de certains risques depuis les années 1990 ; cette année est effectivement reconnue comme étant le début d'une période où le réchauffement s'est accéléré plus significativement.

Enfin, nous avons utilisé les données météorologiques prédites par les modèles de Météo France ou du Cerfacs jusqu'en 2099 sous différents scénarios d'émission afin d'apporter des éléments quant à l'ordre de grandeur de ces évolutions. Ces simulations sont effectuées sur un nombre restreint de stations.

\section{Modèles utilisés}

\section{Le développement}

L'objectif est de mesurer les risques durant des phases de développement de la plante. Une partie de l'approche impose donc la mise en œuvre de modèles capables de simuler les dates d'apparition des principaux stades, notamment la levée, le stade épi à $1 \mathrm{~cm}$, la méiose pollinique, l'épiaison, la floraison et la maturité physiologique. Les modèles employés ont été développés et paramétrés pour les cultivars présents en France [1, 2]. Ces modèles font intervenir la notion de temps vernalophotothermique: I'action journalière de la température sur la vitesse de développement est modulée par un frein photopériodique (le blé est une plante préférentielle de jour long) et par un nombre de jours vernalisants à satisfaire (la plupart des variétés sont vernalisables). L'erreur moyenne de ces modèles est de l'ordre de 5 jours pour le stade épi à $1 \mathrm{~cm}$ et de 3 jours pour l'épiaison.

\section{Les lois d'action du climat}

Plusieurs types d'événements sont calculés.

On détermine fréquentiellement l'occurrence de phénomènes extrêmes à des stades ou à des phases clés de forte sensibilité. Par exemple, la probabilité de rencontrer des gelées (Tmin $<-4{ }^{\circ} \mathrm{C}$ sous abri) après le stade épi à $1 \mathrm{~cm}$, des températures extrêmes préjudiciables (Tmin $<4, \operatorname{Tmax}>32^{\circ} \mathrm{C}$ ) autour de la méiose pollinique et de la fécondation. On calcule également un indice de gel basé sur le cumul des cycles gel-dégel pendant la période automnale et hivernale, en relation avec les dégâts induits sur les céréales. Sur la phase finale du remplissage des grains, sont déterminés le nombre de jours où la température maxi dépasse $25^{\circ} \mathrm{C}$.

Le déficit hydrique est calculé selon un modèle à 2 compartiments (notion de réserve facilement utilisable et de survie) décliné de Choisnel [3]. Comparativement à ce dernier, le modèle développé par ARVALIS détermine une progression journalière du coefficient cultural en fonction des stades et des températures efficaces cumulées. L'état initial de la réserve est fixé à 0 au $1^{\text {er }}$ août avant les semis. Ce modèle permet de calculer le déficit hydrique cumulé (somme des ETR-ETM) sur deux périodes : la période montaison, phase de mise en place du nombre de grains/m2 et celle du remplissage des grains en matière sèche.

Concernant le rendement, nous nous intéressons à deux composantes du rendement : La production du nombre de grains $/ \mathrm{m}^{2}$ et le poids des grains. Pour le nombre de grains, est analysé l'impact du déficit hydrique tout d'abord sur la production de matière sèche à la floraison, en particulier sur la perte relative consécutive à un déficit hydrique cumulé pendant la phase de montaison. Le fait de travailler en valeur relative permet de prendre en charge le fait que les variétés ne requièrent pas le même niveau de biomasse à ce stade pour produire un peuplement en grains non limité. Des lois empiriques ont été établies et sont capables d'établir la relation entre déficit hydrique perte de biomasse - pertes de grains $/ \mathrm{m}^{2}$ [4]. Les pertes de poids de 1000 grains reposent sur la notion de poids de 1000 grains potentiellement accessible par variété, sans facteur limitant autre que celui exercé par le nombre de grains/m2. Les pertes (exprimées en $\mathrm{g}$ ) sont estimées par une régression linéaire combinant l'effet des fortes températures (Tmax seuil $>25^{\circ} \mathrm{C}$ ) et du déficit hydrique cumulé durant cette période [1].

Les différentes simulations ont été faites avec des variétés relativement précoces (de type Soissons et Trémie), et tardives (type Shango). Quelques simulations complémentaires ont été établies avec une orge d'hiver (type de précocité Esterel) dans le but d'introduire un profil phénologique plus précoce, n'existant pas actuellement chez le blé.

Par ailleurs, les calculs mettant en jeu le déficit hydrique ont exécuté en considérant deux niveaux de réserves utiles: $150 \mathrm{~mm}$ pour caractériser les sols profonds et $70 \mathrm{~mm}$ pour représenter les sols superficiels.

\section{Résultats}

\section{L'anticipation des stades}

\section{Climat historique}

En utilisant nos modèles d'apparition des stades sur l'ensemble des années et des stations, on retient qu'en moyenne les dates de réalisation des stades épi à $1 \mathrm{~cm}$ et de l'épiaison ont été avancées en moyenne d'une semaine. Les dates de récolte sont quant à elles plus précoces d'un peu moins de 10 jours. Ces simula- tions sont cohérentes avec les observations pluriannuelles des stades.

Ces valeurs moyennes sont à moduler en fonction des types variétaux : pour les variétés très hiver et sensibles à la durée du jour (type Shango), I'avance s'avère un peu plus faible et au contraire plus forte sur les variétés plus alternatives (type Trémie ou Soissons). Ces chiffres moyens masquent aussi une forte variation interannuelle, l'année 2007 étant avec 1995 la plus précoce au stade épi à $1 \mathrm{~cm}$ avec une avance moyenne de l'ordre de 20 jours.

\section{Climat futur}

En prenant des variétés précoces semées début octobre, on note que l'anticipation des stades sera très significative entre 2070 et 2100 . L'anticipation semble linéaire avec une avancée de 3-4 jours par pas de 20 ans, en moyenne.

En moyenne générale, on peut retenir que le stade épi à $1 \mathrm{~cm}$ sera plus précoce d'environ 25 à 30 jours, en année médiane, en 2100 . II serait ainsi atteint : début février dans les régions du Sud, vers le 10 février en Poitou Charente, Pays de Loire, Bordures maritimes de l'Ouest et du Nord-Ouest, vers le 15 février en lle-de-France, et aux alentours du 22 février en Bourgogne, Champagne et Lorraine. II ne s'agit que de références médianes, ce qui signifie que certaines années, l'avancée de ce stade pourrait être encore bien plus précoce.

Pour l'épiaison, une avancée médiane de 15 à 20 jours peut être retenue. Les références médianes de la date d'épiaison se situeraient entre le 10 et le 20 avril dans les régions du Sud-Est, entre le 20 et 25 avril dans le SudOuest, vers le 25 avril en Poitou-Charentes et Pays de Loire, fin avril ou tout début mai dans les bordures maritimes et en Bretagne, tout début mai en Ile-de-France, et enfin vers le 8 mai dans les régions du Centre-Est.

\section{La croissance}

\section{Matière sèche floraison (nombre de grains $/ \mathrm{m}^{2}$ ) avec le climat historique}

Concernant, la production de grains et la sécheresse en cours de montaison, on constate des évolutions largement fonction du contexte géographique. À proximité des bordures maritimes des régions du nord de la France (Normandie, Bretagne, Picardie, etc.), il n'y a pas eu d'évolution significative : le risque et l'intensité des sécheresses entre le stade épi à $1 \mathrm{~cm}$ et la floraison y sont toujours faibles, avec des pertes nulles en année favorable et normale et, comprise entre 5 et $10 \%$ en année sèche (tableau 1).

Pour l'ensemble des régions du Centre et en périphérie (Région Centre, Bourgogne, Champagne, île-de-France, Pays-de-Loire...), le risque de sécheresse en cours de montaison s'est 
Tableau 1. Évolution comparée des pertes de matière sèche à la floraison (en \%), consécutive au déficit hydrique cumulé en cours de montaison.

\begin{tabular}{|llll|}
\hline $\begin{array}{l}\text { Région (station) pour } \\
\text { une RU de 150 } \mathbf{~ m m}\end{array}$ & $\begin{array}{l}\text { Années } \\
\text { «pluvieuses } \\
\text { (décile 2) }\end{array}$ & $\begin{array}{l}\text { Années normales } \\
\text { (médiane) }\end{array}$ & $\begin{array}{l}\text { Années } \\
\text { "sèches \# } \\
\text { (décile 8) }\end{array}$ \\
\hline Bordure maritime (ex Caen) & & & 5 \\
\hline série 1955-1980 & 0 & 1 & 7 \\
\hline série 1981-2005 & 0 & 1.5 & 8 \\
\hline Région Centre (ex Borges) & & 2 & 20 \\
\hline série 1955-1980 & 0 & $4-6$ & \\
\hline série 1981-2005 & 0 & & 20 \\
\hline Région Sud (ex St Auban) & & 2 & 30 \\
\hline série 1955-1980 & 0 & 8 & \\
\hline série 1981-2005 & 1 &
\end{tabular}

accru de manière significative depuis 25 ans : si en année normale, l'augmentation reste assez marginale, elle s'intensifie très nettement en profils d'années plus « sèches », avec des écarts de l'ordre de $15 \%$ pour des sols dont la réserve utile est assez profonde (tableau 1). Pour des sols plus superficiels, les pénalités sont encore plus nettes, ce qui signifie que pour les petites terres de ces différentes régions concernées (argilo-calcaire de la Bourgogne, du Barrois, du Berry...), la perte de grains $/ \mathrm{m}^{2}$ due à une sécheresse de montaison pourrait devenir une préoccupation pour des surfaces plus grandes. Dans les régions plus au sud et notamment les régions méditerranéennes, on observe y compris en sols profonds, un accroissement plus généralisé du risque de sécheresse pendant la montaison. En année favorable, le risque de sécheresse peut être désormais présent, en année normale, le risque déjà élevé entre 1955 et 1980 s'est intensifié (entre 5 et $10 \%$ ) pour atteindre une élévation de $20 \%$ en années " sèches » (tableau 1). Ainsi en année défavorable, la perte de croissance imputable au manque d'eau se situe à hauteur de $25 \%$. Dans les sols à plus faible réserve, ces pénalités en années difficiles avoisinent $50 \%$, soit une production de grains réduite de moitié avec un tel scénario.

\section{Poids de 1000 grains avec le climat historique}

Nombre de jours échaudants

Le nombre de jours où la température maximale excède $25^{\circ} \mathrm{C}$ entre l'épiaison et la maturité physiologique a augmenté significativement depuis 25 ans malgré une avancée réelle de la date d'épiaison. Toutefois, à l'instar des risques de sécheresse en cours de montaison, cette évolution met en évidence des comportements différents selon les contextes géographiques.

Là où les risques étaient les plus bas entre 1955 et 1980, l'évolution est restée la plus faible: ainsi dans les bordures maritimes du nord et du nord-ouest, le risque initial moyen compris entre 2 et 5 jours selon les stations météoroloentre 2 et 7 jours.

C'est dans les régions du Centre et de sa périphérie décrite plus haut que l'augmentation s'est avérée la plus nette. Selon les stations, le nombre de jours échaudants est effectivement passé d'un intervalle initial de 5 à 11 à un intervalle de 8 à 16 jours selon les stations.

Dans les zones déjà caractérisées par un risque élevé, l'évolution a également augmenté. Dans le Sud-Ouest, en région Rhône-Alpes, le risque est passé de 15-17 à 20-23 jours; En région PACA, le risque moyen de 20-23 jours est dorénavant de 24-27 jours.

\section{Pertes de poids de 1000 grains}

liées à l'échaudage et à la sécheresse

Pour les mêmes régions situées dans les bordures maritimes et proches, il n'y a pas eu d'évolution significative : le risque et l'intensité des sécheresses après la floraison y sont toujours faibles, et c'est toujours la campagne 1976 qui détient de loin le record de sécheresse.

Pour les régions du Centre et de sa périphérie, les pertes moyennes de poids de 1000 grains ont augmenté de 2 à $3 \mathrm{~g}$ en année favorable, de 3 à $4 \mathrm{~g}$ en année normale et sont du même ordre de grandeur en année défavorable.

Pour les régions plus au sud, on constate une élévation des pénalités de 3 à $4 \mathrm{~g}$ quel que soit le profil de l'année.

Ces niveaux de pertes constituent des valeurs significatives puisque, $1 \mathrm{~g}$ de poids de 1000 grains correspond à environ $2 \mathrm{q} /$ ha dans les régions du centre et à environ 1,5 q/ha dans les situations plus au sud.

\section{Les projections pour le futur}

On retrouve la même typologie géographique qu'avec les données historiques. Mais il est important de souligner que les impacts dépendent de manière prépondérante du scénario climatique choisi.

À titre d'exemple, pour la station de Toulouse, le risque médian de rencontrer des températugiques est passé à une fourchette comprise res échaudantes au cours du remplissage avec le scénario A2 s'accroît de 10 jours en 2100 , soit une augmentation d'un peu plus de $50 \%$. En revanche, en considérant un scénario plus optimiste (B2), cette élévation du nombre de jours est beaucoup plus marginale, de l'ordre de 3 ou 4 jours. Avec le scénario A2, les simulations faites sur les pertes de MS au stade floraison révèlent qu'elles restent faibles dans les régions des bordures maritimes du nord et limitrophes. À partir des régions du Centre et plus au sud, on constate une évolution marginale des pénalités jusqu'en 2040 environ, nettement plus soutenues après 2050 , avec un très fort effet de la réserve utile.

Pour le poids des grains, le type d'évolution en fonction des années s'avère identique : faible jusqu'en 2049 puis plus soutenu. Contrairement à la composante précédente, les sols profonds ne sont pas épargnés compte tenu de la forte augmentation des températures excessives au cours du remplissage et de son impact sur le poids de grains. Après 2070, l'évolution semble s'atténuer, plus nettement pour les cultivars les plus précoces via un décalage plus prononcé des stades tardifs. Malgré tout, on note durant cette dernière période une amplification de la variabilité interannuelle.

\section{Voies d'adaptation}

\section{Évitement et phénologie : que faire aujourd'hui}

Depuis 25 ans, le risque de gel d'épis a évolué différemment selon les régions (Gate, 2007). Dans les secteurs des bordures maritimes du nord de la France marquées par l'influence maritime (Normandie, Pays de Loire...), le risque initial déjà élevé s'est accru car l'avancée des stades a été sensible. Plus au Sud, les stades déjà plus précoces font que le risque élevé se maintient, sans évolution. Plus au Centre, les stades étant plus tardifs, le risque initial plus faible reste plus modéré. Plus à l'Est, cette tendance à la tardiveté étant plus marquée, le risque quasi nul reste actuellement marginal. Les projections dans le futur établies sur des stations représentatives de différents types de climat vont toutes dans le même sens : le risque de gel d'épis post-épi à $1 \mathrm{~cm}$ diminuerait si bien que des semis plus précoces seront potentiellement possibles avec un risque plus faible qu'actuellement.

Le risque de gel hivernal tel que nous l'analysons a également diminué depuis 25 ans et continuerait à diminuer partout. Toutefois, le fait que la température automnale et hivernale soit en plus douce pourrait constituer un handicap pour les variétés relativement sensibles car ne permettant un endurcissement suffisant. Depuis 25 ans le risque d'avoir des températures trop basses ou trop élevées à des stades clés 


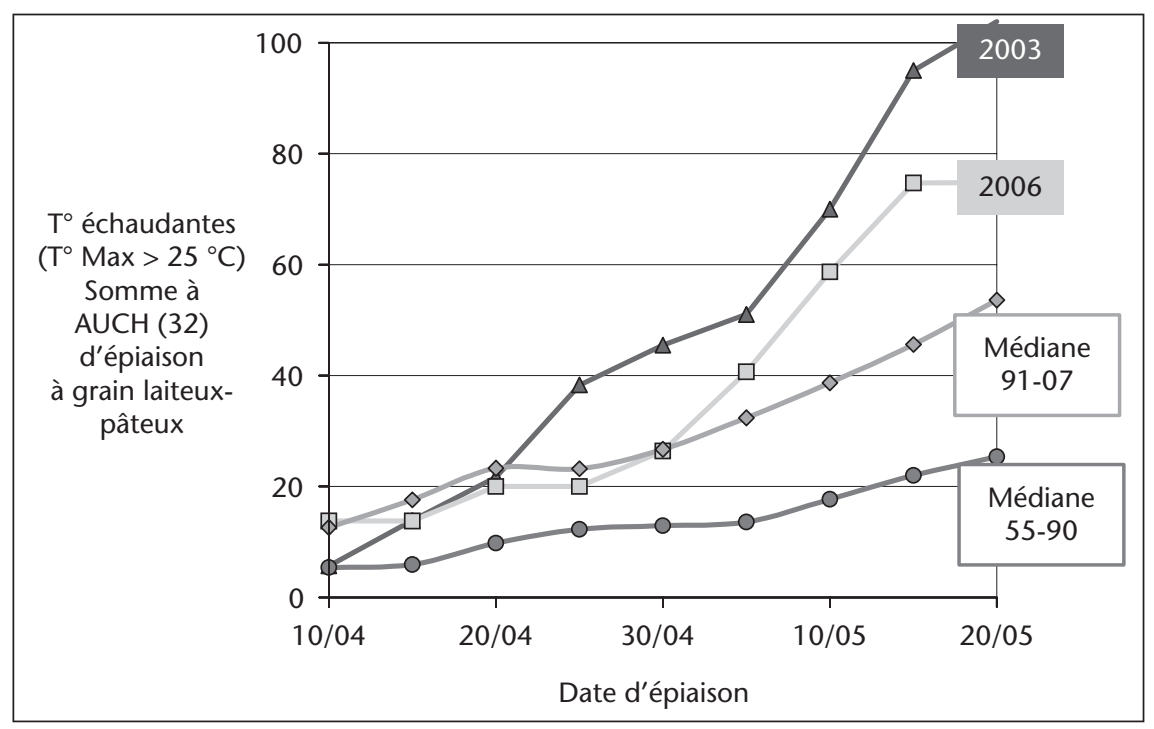

Figure 1. Évolution des températures excédant $25^{\circ} \mathrm{C}$ au cours du remplissage des grains (somme des Tmax $>25^{\circ} \mathrm{C}$ ) en fonction de la date d'épiaison. (exemple de Auch, Gers). Médiane calculée sur 2 séries climatiques et évolution pour 2 années "chaudes", 2003 et 2006.

(méiose, fécondation) n'a été modifié. Leur caractère fortement aléatoire ne permet pas la mise en place d'une stratégie d'évitement efficace.

Ces références et le fait les dégâts occasionnés par le gel d'épi sont souvent partiels et compensables, incitent à mettre la priorité sur l'évitement des stress de fin de cycle.

Hormis quelques régions, I'anticipation des stades s'est avérée et s'avérera insuffisante pour maintenir un niveau identique des risques relatifs aux stress finaux agissant sur le nombre de grains et le poids des grains.

L'analyse des campagnes céréalières montre que le risque d'échaudage thermique s'accroît en fonction de la date d'épiaison (figure 1).

Ces constats prêchent en la faveur d'obtenir des dates d'épiaison précoces dans la plupart des régions.

Dans le cadre des bordures du nord, il est tout à fait possible de ne rien modifier puisque les risques climatiques y sont les plus faibles et $a$ priori peu concernés par l'évolution climatique. Dans les autres régions, la recherche d'une épiaison précoce doit tenir compte de l'augmentation du risque de gels d'épis.

Mais renforcer la présence de variétés précoces ne doit pas engendrer l'utilisation de semis trop précoces : I'attitude est plutôt de semer des variétés précoces à des dates déjà pratiquées sur des variétés plus tardives, en acceptant donc pour la plupart des régions un risque de gel d'épis supérieur.

Effectivement, le recours à des semis ultraprécoces se traduirait par une augmentation plus significative des gels d'épis ainsi que par une élévation plus significative d'autres risques indirects susceptibles d'affecter le coût de pro- duction : en particulier des problèmes d'ordre sanitaire comme les pucerons à l'automne, une infestation plus grande de mauvaises herbes, une augmentation de la quantité d'inoculum «à la sortie de l'hiver » de certaines maladies cryptogamiques (Septoriose et Piétin Verse), et du risque de verse.

Pour les régions caractérisées par un fort échaudage et des sols à faibles réserves (Bourgogne, Berry...), la substitution du blé par l'orge $d^{\prime}$ hiver peut être une stratégie efficace.

\section{Évitement et phénologie: que faire demain}

À l'exception des bordures maritimes du nord, l'adaptation naturelle via l'anticipation des stades n'est pas suffisante. Ceci est illustré par des simulations faites sur la station de Toulouse avec un profil de variété précoce et le scénario A2 (tableau 2).

II convient donc de concevoir des variétés nouvelles plus précoces à l'épiaison. Toutefois, les variétés actuelles de blé tendre les plus précoces à ce stade (de type printemps) sont toutes ultra-précoces au stade épi à $1 \mathrm{~cm}$ et peu ou insensibles à la durée jour.

En cas de scénario avec des températures élevées en automne et en hiver, la date du stade épi à $1 \mathrm{~cm}$ risque donc d'être beaucoup trop précoce avec ce type de variété. La construction de variétés plus précoces à l'épiaison mais néanmoins stable au stade épi à $1 \mathrm{~cm}$ est envisageable en combinant forte précocité intrinsèque et forte exigence en durée du jour. Ces notions font effectivement appel à des gènes de développement que l'on peut introduire au sein d'une même variété.

\section{Caractéristiques de croissance et tolérance variétale}

Certains éléments convergent pour conclure que la biomasse acquise à la floraison risque d'être pénalisée : élévation de la température en cours de montaison, recours à des variétés plus précoces et intensité des déficits hydriques plus soutenue.

II apparaît donc logique de rechercher des variétés à fortes fertilités, c'est-à-dire produisant un effectif élevé de fleurs et également requérant moins de biomasse à la floraison pour produire un nombre de grains $/ \mathrm{m} 2$ suffisant. De telles variétés existent (Trémie, par exemple) et des QTL spécifiques de la fertilité ont d'ores et déjà été identifiées.

Le comportement des variétés au cours du remplissage est en tendance plus stable pour celle possédant un potentiel plus élevé de poids de 1000 grains [1, 4].

Concernant la performance des variétés sous contraintes hydriques, nos résultats ont montré qu'elle est en relation étroite avec l'efficience d'utilisation de l'eau, quintaux de grains produits par mm d'eau consommé $[4,5]$. La consommation d'eau, difficilement accessible avec suffisamment de précision à l'échelle du champ, peut être estimée indirectement par le dosage des isotopes naturels du carbone photosynthétique du grain $[4,6]$.

Comme les stress abiotiques et biotiques sont susceptibles de coexister dans une même parcelle, des méthodes visant à identifier la tolérance globale des variétés, basée sur la notion d'indice foliaire vert intégré au cours du remplissage, sont actuellement en cours d'évaluation [7].

\section{Autres techniques culturales}

Dans les milieux séchants, il est recommandé d'élargir la gamme de précocité des semis afin de pouvoir profiter de conditions plus favora-

Tableau 2. Évolution des dates de stade pour maintenir les pertes de poids des grains à un niveau équivalent à celui d'aujourd'hui. Ex. station de Toulouse, RU de $150 \mathrm{~mm}$ et précocité type Trémie.

\begin{tabular}{|llll|}
\hline Année de semis & Aujourd'hui & $\mathbf{2 0 3 0}$ & $\mathbf{2 0 6 0}$ \\
\hline Date d'épiaison & 5 mai & $1^{\text {er }}$ mai & 23 avril \\
\hline $\begin{array}{l}\text { Date d'épiaison pour maintenir un même niveau } \\
\text { de risque }\end{array}$ & 20 avril & 10 avril \\
\hline Soit un stade épi à $1 \mathrm{~cm}$ & 10 mars & 25 février & 15 février \\
\hline
\end{tabular}


bles à la germination et à l'émergence. En années plus sèches, il convient de préférer les préparations du sol simplifiées afin de mieux conserver l'état d'humidité et de ne pas rompre les remontées capillaires possibles, et également de semer plus profond. Pour certaines implantations de printemps, le recours à des cultures intermédiaires (légumineuses) a fait ces preuves (meilleure répartition de l'azote et structure favorable à un enracinement rapide). Vis-à-vis de la gestion de la fertilisation azotée, les constats établis montrent une tendance à une minéralisation précoce favorisée (imputable à des températures plus douces, une disponibilité en eau sur cette période). À l'opposé, I'analyse des essais révèle une plus forte irrégularité du coefficient de valorisation des engrais $(C A U)$, en relation étroite avec le déficit hydrique ou pluviométrique en cours de montaison. En région sud, et plus généralement en sols légers, cette irrégularité est renforcée par des fins de cycle avec des stress abiotiques plus fréquents. II convient donc que la gestion de l'azote intègre avec les outils de pilotage, les prévisions météorologiques (pour le CAU) et modèles de prévision du rendement accessible. Pour la verse, il est probable que le risque potentiel en début de montaison soit en tendance plus élevé par un tallage plus abondant (températures reçues au cours du tallage, plus élevées eau disponible), minéralisation précoce favorisée et également montée des tiges en jours plus courts.

\section{Conclusion}

On conclut à des évolutions différenciées selon les contextes géographiques : les réflexions et les solutions que nous proposons ne sont donc pas identiques; leur mise en œuvre se fera de ce fait selon un calendrier distinct, fonction de l'impact plus ou moins fort et rapide du climat sur la production.

Un travail parallèle montre par ailleurs que la variabilité interannuelle du rendement après les années 1990 est expliquée en moyenne pour différentes régions, à hauteur de $65 \%$ par les conditions climatiques que nous avons analysées : déficit hydrique et échaudage thermique, notamment. Ces constats sont convergents avec des travaux menés à l'échelle mondiale [8]. Ces auteurs mettent effectivement en évidence que depuis les années 1990, le rendement et la production du blé ont été affectés par l'élévation de la température et de la sécheresse. Une autre étude conduite sur le riz, autres céréales en $\mathrm{C} 3$, conclut que les températures de nuit ont constitué le facteur le plus limitant du rendement $[9,10]$. Ce fait témoigne de la contribution de l'augmentation de la respiration dans les pertes de rendement, indépendamment de la photorespiration ou de la sécheresse.

En France, dans les régions où l'échaudage et la sécheresse de fin de cycle sont présents (comme en Bourgogne, dans le Berry), on constate que l'augmentation annuelle du rendement après les années 1990 s'infléchit moins pour l'orge d'hiver que pour le blé tendre, compte tenu de sa plus grande précocité. Ces constats sont cohérents avec notre analyse.

Nos résultats montrent effectivement l'importance de la phénologie pour atténuer l'impact des risques. Mais globalement, si l'on se projette dans le futur avec un scénario assez pessimiste, on peut retenir que l'anticipation naturelle fait en moyenne la moitié du chemin (risque d'échaudage). Maintenir dans le futur un niveau équivalent à celui d'aujourd'hui impose la construction de nouveaux profils phénologiques, en profitant des atouts génétiques des céréales. Ces nouveaux profils sont à définir en poursuivant l'analyse sur un plus grand nombre de stations et de risques. La poursuite de telles études se fait actuellement dans le cadre d'un projet de recherche sur la plupart des espèces cultivées (projet ANR Climator).

D'une manière générale, nos simulations avec les données historiques et futures mettent en avant les préjudices occasionnés par l'augmentation des fortes températures. Ces derniers intéressent effectivement la plupart des bassins de production et tous types de sol.

Ce point est à mettre en relation avec une limite de notre étude qui ne prend pas en compte les effets possibles de compensation de l'élévation de la teneur en $\mathrm{CO}_{2}$. En effet, une augmentation significative de cet élément devrait réduire théoriquement la sensibilité des céréales aux fortes températures. Néanmoins, les références acquises en doublant la concentration de cet élément à l'échelle du champ concluent à un effet mitigé [11]. De telles conclusions prêchent en la faveur d'un renforcement des recherches sur la tolérance à la canicule et plus largement sur les interactions entre excès de température, sécheresse et taux de $\mathrm{CO}_{2}$. Les fortes températures ont effectivement une action directe sur la fermeture des stomates, les phénomènes de respiration nocturnes et l'activité des enzymes dont les amylases synthases.

Enfin, avec une anticipation importante des stades, une variabilité climatique qui semble $s^{\prime}$ amplifier, on ne peut rejeter l'hypothèse au moins d'un maintien des risques au moment de la méiose et de la fécondation, au moins dans certains types de milieux. Ceci met l'accent sur I'intérêt de caractériser l'aptitude des variétés à franchir ces étapes cruciales sous une plus large gamme de conditions environnementales.

Remerciements. Nadine Brisson (INRA); Philippe Braun (Arvalis); Météo France et le Cerfacs.

\section{RÉFÉRENCES}

1. Gate P. Ecophysiologie du blé : de la plante à la culture. Paris : Lavoisier Editeur, 1995.

2. Brisson N, Ruget F, Gate P, et al. STICS : a generic model for the simulation of crops and their water and nitrogen balances. II- Model validation for wheat and maize. Agronomie $2002 ; 22$ : 69-92

3. Choisnel $E$. Un modèle agrométéorologique opérationnel de bilan hydrique utilisant des données climatiques. In : Conf. Int. CIID. Les besoins en eau des cultures. Paris: éd. INRA, 1985 : 115-32.

4. Gate P. Dossier «le blé face au changement climatique ». Perspectives Agricoles 2007(336) : 20-56.

5. Gate P, Bouthier A, Casabianca H, Deléens E. Caractères physiologiques décrivant la tolérance à la sécheresse du blé cultivé en France : interprétation des corrélations entre le rendement et la composition isotopique du carbone des grains. Séminaire «Tolérance à la sécheresse des céréales en zone méditerranéenne. Diversité génétique et amélioration variétale ». Montpellier, 15-17 décembre 1992.

6. Deléens E, Hannachi L, Gate P, Casabianca H. Composition isotopique du carbone du blé au cours du développement : efficacité d'utilisation de l'eau de la plante et des différents organes verts participant au remplissage. In : Maillard P, Bonhomme R, eds. Utilisation des isotopes stables pour l'étude du fonctionnement des plantes. INRA publications, $1995:$ 65-82.

7. Gate P, Gouache D, Robert C, Bancal MO, Lannou C, Ney B. Quel indicateur plante pour rendre compte de la nuisibilité de la septoriose sur blé tendre? Actes de la CIMA 2006.

8. Lobell DB, Field CB. Global scale climate-crop yield relationships and the impacts of recent warming. Environ Res Let 2007(2) : 14002.

9. Peng S, Huang J, Sheehy J, et al. Rice yields decline with higher temperature from global warming. Agriculural Sciences, PNAS 2004; $101: 9971-5$.

10. Collectif. Coup de chaud sur le riz. Le Monde ; 27/04/2004.

11. Long S, Ainsworth E, Leakey A, Morgan P. Global food insecurity. Treatment of major foods crops with elevated carbon dioxide or ozone under large-scale fully open-air conditions suggests recent models may have overestimated future yields. Phil Trans R Soc B 2005 ; 360 : 2011-20. 